\title{
Model-Following Designs Using Direct State Derivative Measurement Feedback in Novel Reciprocal State Space Form
}

\author{
Yuan-Wei Tseng, Rong-Ching $\mathrm{Wu}^{*}$ \\ Department of Electrical Engineering, I-Shou University, Taiwan \\ Email: yuanwei@isu.edu.tw, ${ }^{\star}$ rcwu@isu.edu.tw
}

How to cite this paper: Tseng, Y.-W. and Wu, R.-C. (2019) Model-Following Designs Using Direct State Derivative Measurement Feedback in Novel Reciprocal State Space Form. Journal of Applied Mathematics and Physics, 7, 394-409.

https://doi.org/10.4236/jamp.2019.72030

Received: January 24, 2019

Accepted: February 22, 2019

Published: February 25, 2019

Copyright $\odot 2019$ by author(s) and Scientific Research Publishing Inc. This work is licensed under the Creative Commons Attribution International License (CC BY 4.0).

http://creativecommons.org/licenses/by/4.0/

\begin{abstract}
The paper introduces effective and straightforward algorithms of both explicit and implicit model-following designs with state derivative measurement feedback in novel reciprocal state space form (RSS) to handle state derivative related performance output and state related performance output design cases. Applying proposed algorithms, no integrators are required. Consequently, implementation is simple and low-cost. Simulation has also been carried out to verify the proposed algorithms. Since acceleration can only be modeled as state derivative in state space form and micro-accelerometer which is the state derivative sensor is getting more and more attentions in many microelectromechanical and nanoelectromechanical systems (MEMS/NEMS) applications, the proposed algorithms are suitable for MEMS/NEMS systems installed with micro-accelerometers.
\end{abstract}

\section{Keywords}

Reciprocal State Space Form, State Derivative Measurement Feedback, Explicit Model-Following Design, Implicit Model-Following Design

\section{Introduction}

In the past, the approach of expressing system in state space form and applying state measurement feedback was popular in developing many effective control algorithms as well as design tools for its strong abilities to deal with multi-input multi-output (MIMO) systems. However, the combination of expressing systems in state space form and applying state related feedback still has its limitations in control designs because in practical, not every system can have its state space form or can measure state related signals for feedback design. For example, 
when systems have open loops at infinity, they are categorized as singular systems [1] and cannot be expressed in state space form. Therefore, state design approach cannot handle singular systems. There are quite a few singular systems that people encounter in particle designs such as electrical networks [2], aerospace systems [3], smart structures [4] and chemical processes [5]. Furthermore, in many applications, the system sensors directly measure state derivative signals rather than state signals. For such case, it is better to apply state derivative related feedback signals than state related feedback signals in control design to avoid using integrators which can increase cost and complexity of controller implementation.

For example, accelerometers [6] that measure accelerations are popular sensors in many structural and vibration control applications. Considering a dynamic second order system with acceleration measurement feedback in control design as follows

$$
\begin{gathered}
M \ddot{q}+D \dot{q}+K q=b u \\
y=c \ddot{q} \\
u=-k y
\end{gathered}
$$

where $q$ is the displacement vector while $u$ is the control input vector.

Defining $x=\left[\begin{array}{ll}q & \dot{q}\end{array}\right]^{\mathrm{T}}$, the system can be modeled in the following state space form.

$$
\begin{gathered}
\dot{x}=\left[\begin{array}{c}
\dot{q} \\
\ddot{q}
\end{array}\right]=\left[\begin{array}{cc}
0 & I \\
-M^{-1} K & -M^{-1} D
\end{array}\right]\left[\begin{array}{l}
q \\
\dot{q}
\end{array}\right]+\left[\begin{array}{c}
0 \\
M^{-1} b
\end{array}\right] u=A x+B u \\
y=\left[\begin{array}{ll}
0 & c
\end{array}\right]\left[\begin{array}{l}
\dot{q} \\
\ddot{q}
\end{array}\right]=C \dot{x} \\
u=-k y=-k C \dot{x} \\
\dot{x}=(I+B k C)^{-1} A x
\end{gathered}
$$

However, mathematically speaking, state space form is not suitable to carry out state derivative related feedback design. In state space form, the highest order signal, acceleration $\ddot{q} \mathrm{~s}$ always belong to state derivative $\dot{x}$. From the closed loop system in (2d), one can easily understand that state derivative measurement feedback designs cannot be carried out as straightforward as state measurement feedback designs in state space form because the state derivative feedback gain $\mathrm{k}$ is coupled with many matrices and inside an inverse. Consequently, complex and advanced mathematics are needed to design state derivative feedback control algorithms. If the direct measurement of a system is state derivative related, to apply the control algorithms based on state related feedback design, additional integrators or numerical integrations are required and consequently result in both high cost and complexity in implementation. In the past, the majority of design approaches that handled singular systems and state derivative measurement feedback control used the following generalized state space form [7]. 


$$
E \dot{x}=F x+N u
$$

Mathematically speaking, the available control design algorithms are usually carried out in augmented systems of large size and require feedbacks of both state and state derivative variables for generalized state space systems [8] [9] [10] [11] [12]. They are much more complex than those for the standard state space form. Especially, singular systems are further categorized as impulse-free ones [13] and with impulse mode ones [8] in analysis. If a singular system has impulse mode, further investigations of impulse controllable and the impulse mode elimination [8] have to be analyzed in control designs. Therefore, singular systems with impulse mode are usually considered as difficult cases in control designs.

To provide supplementary design algorithms for systems which cannot be modeled in standard state space form and to utilize direct state derivative measurement feedback in control designs in similar ways as state measurement feedback control designs in standard state space form, the following novel reciprocal state space (RSS) form was proposed [14] [15]. When the matrix $F$ in (3) is non-singular (full rank) and only state derivatives are directly measured and fed back, the system can be expressed in reciprocal state space (RSS) form as follows.

$$
\begin{gathered}
x=F^{-1} E \dot{x}-F^{-1} N u=A \dot{x}+B u \\
y=C \dot{x} \\
u=-K y=-K C \dot{x}
\end{gathered}
$$

The name of reciprocal state space (RSS) form was given because the eigenvalues of $A$ in (4a) are the reciprocals of the eigenvalues of the open loop system. From (4a), for an open loop system which has no pole at zero but can have pole at infinity can be expressed in RSS form. Therefore, some singular systems can be handled in RSS form. The controllability and observability analyses for system in RSS form have been investigated in [14] [16]. It showed that they turn out to be the same as their counterparts in state space form. It also showed that applying state derivative feedback in RSS form can easily carry out some popular control design techniques such as pole placement, eigenstructure assignment, and linear quadratic regulator (LQR) [14] [17]. Note that some singular systems with impulse mode can also be expressed in RSS form to easily carry out state derivative measurement feedback control designs [15] [17].

Model-following designs are LQR techniques to utilize output feedback controllers so that the system's performance output can behave as close to a desired model's performance output as possible. Although the closed loop system may not perform exactly the same as the desired model, the closed loop system is asymptotically stable. Therefore, through the model-following designs, at least the stability of closed loop system is guaranteed. Implementation simplicity and low cost are the major advantages of such designs because the numbers of sensors and actuators employed in the system can be fewer or much fewer than the number of state variables, especially in structure control applications. State 
measurement based model-following output feedback designs were developed in [18] [19]. Model-following designs are basically categorized into explicit and implicit ones [18]. In explicit model-following design, the model explicitly appeared in the controller as a feed-forward compensator to drive the plant states to zero in such a way that the performance output follows the model output. On the other hand, for implicit model-following design, the model appears in not the control structure but in the performance index. Therefore, explicit model-following is also called model in the system control while implicit model-following is also called model in the performance index control.

In previous studies, model-following designs were carried out in state space form and utilized state measurement. To handle more problems, to increase implementation flexibility in sensor selection and to provide supplementary design algorithms of applying state derivative measurement feedback, in this paper, the designs are carried out in RSS form and utilized state derivative measurement. According to different considerations of performance outputs, explicit model follower in RSS form is proposed when performance outputs are related to state derivatives while implicit model follower in RSS form is proposed when performance outputs are related to states. The proposed model-following design algorithms using state derivative measurement in RSS form are analogous to those in standard state space form using state measurement. Therefore, a lot of mathematic overhead can be saved and more problems can be handled.

\section{Explicit Model-Following Design in Reciprocal State Space Form for State Derivative Related Performance Output Case}

In this section, an explicit model-following control algorithm applying state derivative measurement feedback is proposed when the desired performance output is state derivative related.

A dynamic system is expressed in reciprocal state space (RSS) form as follows.

$$
\begin{gathered}
x^{\prime}=A \dot{x}^{\prime}+B^{\prime} u \\
y^{\prime}=C^{\prime} \dot{x}^{\prime} \\
u=-k y^{\prime}
\end{gathered}
$$

where, $x^{\prime}, y^{\prime}$ and $u$ are state vector, measurement vector and control input vector, respectively.

Substituting (5b)-(5c) into (5a), the corresponding closed loop system is obtained as

$$
x^{\prime}=\left(A^{\prime}-B^{\prime} k C^{\prime}\right) \dot{x}^{\prime}=A_{c} \dot{x}^{\prime}
$$

Please note that the eigenvalues of $A_{c}$ are exactly the reciprocals of eigenvalues of closed loop system. That is why (5) are called reciprocal state space system.

Suppose that the performance output $Z$ that consists of state derivatives is given as follows. 


$$
Z=H \dot{x}^{\prime}
$$

The performance output $Z$ is the most concerned output information which may not be directly measured in the system. Therefore, matrix $H$ in (7) is usually not related to sensor implementation.

The desired model to be followed by the controlled system is given as follows.

$$
\begin{aligned}
& \underline{x}=\underline{A} \underline{\dot{x}} \\
& \underline{y}=\underline{C} \dot{x} \\
& \underline{Z}=\underline{H} \underline{\dot{x}}
\end{aligned}
$$

Note that the eigenvalues of matrix $\underline{A}$ in (8a) should be chosen to be the reciprocals of the desired closed loop eigenvalues. The model mismatch error $e$ is defined as follows.

$$
e=\underline{Z}-Z=\underline{H} \underline{\dot{x}}-H \dot{x}^{\prime}
$$

Therefore, the sizes of matrices $\underline{H}$ and $H$ must be the same while the sizes of matrix $C$ in (8b) and matrix $C^{\prime}$ in (5b) can be different.

By defining the augmented state as $x=\left[\begin{array}{ll}x^{\prime} & \underline{x}\end{array}\right]^{\mathrm{T}}$, one obtain the following augmented system of the original system and model in RSS form.

$$
\begin{gathered}
x=\left[\begin{array}{ll}
A^{\prime} & 0 \\
0 & \underline{A}
\end{array}\right] \dot{x}+\left[\begin{array}{c}
B^{\prime} \\
0
\end{array}\right] u=A \dot{x}+B u \\
y=\left[\begin{array}{l}
y^{\prime} \\
y
\end{array}\right]=\left[\begin{array}{cc}
C^{\prime} & 0 \\
0 & \underline{C}
\end{array}\right] \dot{x}=C \dot{x}
\end{gathered}
$$

To let the controlled system behave like the model, the transient model mismatch error must be minimized. One should properly select symmetric positive definite error weighting matrix $Q^{\prime}$ and control weighting matrix $R$ to define the following performance index $J_{1}$.

$$
J_{1}=\frac{1}{2} \int_{0}^{\infty}\left(e^{\mathrm{T}} Q^{\prime} e+u^{\mathrm{T}} R u\right) \mathrm{d} t
$$

Substituting (9)-(10) into $J_{1}$, we have

$$
\begin{aligned}
J_{1} & =\frac{1}{2} \int_{0}^{\infty}\left[\left(\underline{H} \underline{\dot{x}}-H \dot{x}^{\prime}\right)^{\mathrm{T}} Q^{\prime}\left(\underline{H} \underline{\dot{x}}-H \dot{x}^{\prime}\right)+u^{\mathrm{T}} R u\right] \mathrm{d} t \\
& =\frac{1}{2} \int_{0}^{\infty}\left[(\dot{x})^{\mathrm{T}} Q(\dot{x})+u^{\mathrm{T}} R u\right] \mathrm{d} t
\end{aligned}
$$

where

$$
Q=\left[\begin{array}{cc}
H^{\mathrm{T}} Q^{\prime} H & -H^{\mathrm{T}} Q^{\prime} \underline{H} \\
-\underline{H}^{\mathrm{T}} Q^{\prime} H & \underline{H^{\mathrm{T}}} Q^{\prime} \underline{H}
\end{array}\right]
$$

Since performance outputs in both system (7) and model (8c) could have nothing to do with real implementation, matrices $H$ in (7) and $\underline{H}$ in (8c) are considered as design parameters as shown in $Q$.

Defining $K=\left[\begin{array}{ll}k_{p} & k_{m}\end{array}\right]$ and applying direct output feedback of the augmented system, one obtain

$$
u=-K y=-K C \dot{x}=-k_{p} y^{\prime}-k_{m} \underline{y}=-k_{p} C^{\prime} \dot{x}^{\prime}-k_{m} \underline{C} \underline{\dot{x}}
$$


Thus both the plant output and model output are required. Now, it is clear that the model acts as a compensator in the proposed explicit model-following design with state derivative measurement feedback.

Note that the proposed performance index in (12) is non-traditional because it is not state quadratics but state derivative quadratics.

Assume that $A$ and $B$ in (10) are controllable, the closed loop system becomes

$$
x=(A-B K C) \dot{x}=A_{c} \dot{x}
$$

For a linear time invariant system, it is globally asymptotically stable if the real parts of all system poles are strictly negative. Therefore, such systems have no poles at infinity or zero. Consequently, a globally asymptotically stable system can be expressed in both state space form and RSS form. From system constraint in (15), we can conclude that if closed loop system is asymptotically stable, $A_{c}$ in (15) is invertible and both $x$ and $\dot{x}$ can be expressed by each other. We can also conclude that that both $x$ and $\dot{x}$ must vanish with time when closed loop system is properly controlled and asymptotically stable.

Substituting (14) into (12), the performance index $J_{1}$ becomes

$$
J_{1}=\int_{0}^{\infty} \dot{x}^{\mathrm{T}}\left(Q+C^{\mathrm{T}} K^{\mathrm{T}} R K C\right) \dot{x} \mathrm{~d} t
$$

Suppose that a constant, symmetric, positive semi-definite matrix $P$ can be found such that

$$
\begin{aligned}
J_{1} & =\int_{0}^{\infty} \dot{x}^{\mathrm{T}}\left(Q+C^{\mathrm{T}} K^{\mathrm{T}} R K C\right) \dot{x} \mathrm{~d} t=\int_{0}^{\infty}-\frac{\mathrm{d}}{\mathrm{d} t}\left(x^{\mathrm{T}} P x\right) \\
& =x^{\mathrm{T}}(0) P x(0)-\lim _{t \rightarrow \infty} x^{\mathrm{T}}(t) P x(t)
\end{aligned}
$$

where $x(0)$ is the state vector of initial condition. Since $x(t)$ vanishes with time when closed-loop system is asymptotically stable, $J_{1}$ becomes

$$
J_{1}=x^{\mathrm{T}}(0) \operatorname{Px}(0)
$$

Applying (15), for an asymptotically stable closed loop system, we have

$$
\frac{\mathrm{d}}{\mathrm{d} t}\left(x^{\mathrm{T}} P x\right)=\dot{x}^{\mathrm{T}} P x+x^{\mathrm{T}} P \dot{x}=\dot{x}^{\mathrm{T}}\left(P A_{c}+A_{c}^{\mathrm{T}} P\right) \dot{x}=-\dot{x}^{\mathrm{T}}\left(Q+C^{\mathrm{T}} K^{\mathrm{T}} R K C\right) \dot{x}
$$

Consequently, a constraint is obtained as

$$
g_{1}=Q+C^{\mathrm{T}} K^{\mathrm{T}} R K C+A_{c}^{\mathrm{T}} P+P A_{c}=0
$$

We may write (18) as

$$
J_{1}=\operatorname{tr}(P X)
$$

where $t r$ stands for trace and

$$
X=x(0) x^{\mathrm{T}}(0)
$$

Thus one can adjoin the constraint in (20) to the performance index by defining the Hamiltonian as

$$
H=\operatorname{tr}(P X)+\operatorname{tr}\left(g_{1} S\right)
$$

where $S$ is a symmetric $n \times n$ matrix of Lagrange multipliers and needs to be 
solved. The partial derivatives of $H$ with respect to $P, S$ and $K$ must be equal to zero in order to minimize $J_{1}$. Therefore, the following three coupling equations are necessary conditions for the solution of LQR problem with state derivative measurement feedback

$$
\begin{gathered}
\frac{\partial H}{\partial S}=g_{1}=Q+C^{\mathrm{T}} K^{\mathrm{T}} R K C+A_{c}^{\mathrm{T}} P+P A_{c}=0 \\
\frac{\partial H}{\partial P}=A_{c} S+S A_{c}^{\mathrm{T}}+X=0 \\
\frac{\partial H}{\partial K}=R K C S C^{\mathrm{T}}-B^{\mathrm{T}} P S C^{\mathrm{T}}=0
\end{gathered}
$$

Note that (24) and (25) are well known Lyapunov equations for $P$ and $S$, respectively. Lyapunov equations can be easily solved by many available tools. However, if we stick on state derivative measurement feedback design in state space form and apply closed loop system similar in (2d), we will have much more complex equations and have to spend time to develop tool to solve them. In (26), $R$ is positive definite. When $C S C^{\mathrm{T}}$ is nonsingular, we have

$$
K=R^{-1} B^{\mathrm{T}} P S C^{\mathrm{T}}\left(C S C^{\mathrm{T}}\right)^{-1}
$$

Bear in mind that the closed loop poles are the reciprocals of matrix $A_{c}$ in (15). We can observe that the (24)-(26) used to solve for the optimal state derivative measurement feedback gain $\mathrm{K}$ in (27) are identical to those of a standard state space system with state output feedback to minimize the traditional performance index as follows [18].

$$
J_{2}=\int_{0}^{\infty}\left(x^{\mathrm{T}} Q x+u^{\mathrm{T}} R u\right) \mathrm{d} t
$$

Subject to

$$
\begin{gathered}
\dot{x}=A x+B u \\
y=C x \\
u=-K y
\end{gathered}
$$

Therefore, iterative solution algorithm in [18] can be adopted to write the MATLAB program to solve for the optimal state derivative measurement feedback gain $K$. It is concluded that if the system with state derivative measurement feedback is defined as RSS form, finding LQR measurement feedback gain $K$ becomes straightforward.

\section{Implicit Model-Following Design in Reciprocal State Space Form for State Related Performance Output Case}

In this section, an implicit model-following control algorithm applying state derivative measurement feedback is proposed for a system when its desired performance output is state related.

Suppose that the sensors of a system directly measure state derivatives rather than states and the measurement is directly utilized in feedback design without 
being integrated and the system in RSS form is given as follows.

$$
\begin{gathered}
x=A \dot{x}+B u \\
y=C \dot{x} \\
u=-K y=-K C \dot{x}
\end{gathered}
$$

where $x, y$ and $u$ are state vector, measurement vector and control input vector, respectively.

Substituting (30b) and (30c) into (30a), the corresponding closed loop system is obtained as

$$
x=(A-B K C) \dot{x}=A_{c} \dot{x}
$$

Suppose that the performance output is state related and is given as

$$
Z=H x
$$

Taking derivative of both sides in (32), we have

$$
\dot{Z}=H \dot{x}
$$

To design the RSS implicit model-following controller, an ideal RSS model should be selected as

$$
\underline{Z}=\underline{A \dot{Z}}
$$

where $\underline{Z}$ is the performance output of ideal model.

Note that the eigenvalues of matrix $\underline{A}$ in (34) should be chosen as the reciprocals of the desired closed loop eigenvalues. If the size of matrix $\mathrm{H}$ in (11) is $p \times n, \underline{A}$ in (34) is $p \times p$. Therefore, for a $n \times n$ system, only $p$ ideal eigenvalues are specified in the ideal model in (34).

Applying (30)-(33), model mismatch error $e$ is defined as follows.

$$
e=Z-\underline{A} \dot{Z}=H x-\underline{A} H \dot{x}=H A \dot{x}+H B u-\underline{A H} \dot{x}=(H A-\underline{A H}) \dot{x}+H B u
$$

Regarding (35), the reason why mismatch error is defined as

$$
e=Z-\underline{A} \dot{Z}
$$

rather than the actual error between system and model,

$$
e=Z-\underline{Z}=Z-\underline{A} \underline{\dot{Z}}
$$

is to avoid the use of augmented system in controller design because an ideal model has to be a compensator in controller structure for an augmented system. Therefore, using (35) to define model mismatch error, model will not appear in controller structure. That is the reason why this type of design is called implicit model following design. Although (35) is not the exact mismatch error between system and ideal model, it still makes sense for matrix $\underline{A}$ having the ideal closed loop behavior that should be followed by the system.

The purpose of implicit model-following controller is to minimize the transient model mismatch error and to keep the closed loop system stable.

Therefore the performance index is defined as

$$
J=\frac{1}{2} \int_{0}^{\infty}\left(e^{\mathrm{T}} Q e+u^{\mathrm{T}} R u\right) \mathrm{d} t
$$


Substituting (35) into (38), we have

$$
\begin{aligned}
J & =\frac{1}{2} \int_{0}^{\infty}\left[\left(\dot{x}^{\mathrm{T}} A^{\mathrm{T}} H^{\mathrm{T}}-\dot{x}^{\mathrm{T}} H^{\mathrm{T}} \underline{A}^{\mathrm{T}}+u^{\mathrm{T}} B^{\mathrm{T}} H^{\mathrm{T}}\right) Q(H A \dot{x}-\underline{A} H \dot{x}+H B u)+u^{\mathrm{T}} R u\right] \mathrm{d} t \\
& =\frac{1}{2} \int_{0}^{\infty}\left(\dot{x}^{\mathrm{T}} Q^{\prime} \dot{x}+2 \dot{x}^{\mathrm{T}} W u+u^{\mathrm{T}} R^{\prime} u\right) \mathrm{d} t
\end{aligned}
$$

where

$$
\begin{aligned}
& Q^{\prime}=(\underline{A} H-H A)^{\mathrm{T}} Q(\underline{A} H-H A) \\
& W=(H A-\underline{A} H)^{\mathrm{T}} Q H B \\
& R^{\prime}=B^{\mathrm{T}} H^{\mathrm{T}} Q H B+R
\end{aligned}
$$

Substitute (30c) into (39), the performance index becomes

$$
J=\frac{1}{2} \int_{0}^{\infty}\left[\dot{x}^{\mathrm{T}}\left(Q^{\prime}-2 W K C+C^{\mathrm{T}} K^{\mathrm{T}} R^{\prime} K C\right) \dot{x}\right] \mathrm{d} t
$$

Suppose there exists a constant, symmetric, positive semi-definite matrix $P$ such that

$$
\frac{\mathrm{d}}{\mathrm{d} t}\left(x^{\mathrm{T}} P x\right)=-\dot{x}^{\mathrm{T}}\left(Q^{\prime}-2 W K C+C^{\mathrm{T}} K^{\mathrm{T}} R^{\prime} K C\right) \dot{x}
$$

Then we have

$$
\begin{aligned}
J & =\frac{1}{2} \int_{0}^{\infty}\left[\dot{x}^{\mathrm{T}}\left(Q^{\prime}-2 W K C+C^{\mathrm{T}} K^{\mathrm{T}} R^{\prime} K C\right) \dot{x}\right] \mathrm{d} t \\
& =x^{\mathrm{T}}(0) P x(0)-\lim _{t \rightarrow \infty} x^{\mathrm{T}}(t) P x(t)
\end{aligned}
$$

where $x(0)$ is the state vector of initial condition. Since $x(t)$ vanishes with time when closed-loop system is asymptotically stable, $J$ further becomes

$$
J=x^{\mathrm{T}}(0) \operatorname{Px}(0)
$$

Since

$$
\frac{\mathrm{d}}{\mathrm{d} t}\left(x^{\mathrm{T}} P x\right)=\dot{x}^{\mathrm{T}} P x+x^{\mathrm{T}} P \dot{x}=\dot{x}^{\mathrm{T}} P A_{c} \dot{x}+\dot{x}^{\mathrm{T}} A_{c}^{\mathrm{T}} P \dot{x}=\dot{x}^{\mathrm{T}}\left(A_{c}^{\mathrm{T}} P+P A_{c}\right) \dot{x}
$$

Comparing (44) with (47), one obtains the following constraint equation.

$$
g=A_{c}^{\mathrm{T}} P+P A_{c}+C^{\mathrm{T}} k^{\mathrm{T}} R^{\prime} k C-2 k C W+Q^{\prime}
$$

We may write (46) as

$$
J=\operatorname{tr}(P X)
$$

where

$$
X=x(0) x^{\mathrm{T}}(0)
$$

Thus adjoin the constraint in (48) to the performance index by defining the Hamiltonian as

$$
H=\operatorname{tr}(P X)+\operatorname{tr}(g S)
$$

where $S$ is a symmetric $n \times n$ matrix of Lagrange multiplier and needs to be solved. The partial derivatives of $\mathrm{H}$ with respect to $P, S$ and $K$ must be equal to 
zero in order to minimize $J$. Therefore, the following three coupling equations are necessary conditions for the solution of the LQR problem.

$$
\begin{gathered}
\frac{\partial H}{\partial S}=g=A_{c}^{\mathrm{T}} P+P A_{c}+C^{\mathrm{T}} k^{\mathrm{T}} R^{\prime} k C-2 k C W+Q^{\prime}=0 \\
\frac{\partial H}{\partial P}=A_{c} S+S A_{c}^{\mathrm{T}}+X=0 \\
\frac{\partial H}{\partial K}=R^{\prime} K C S C^{\mathrm{T}}-(P B+W)^{\mathrm{T}} S C^{\mathrm{T}}=0
\end{gathered}
$$

Note that (52) and (53) are Lyapunov equations for $P$ and $S$, respectively. Since $R^{\prime}$ is positive definite and if $C S C^{\mathrm{T}}$ is nonsingular, from (54) there exists the solution of optimal gain as follows.

$$
K=\left(R^{\prime}\right)^{-1}(P B+W)^{\mathrm{T}} S C^{\mathrm{T}}\left(C S C^{\mathrm{T}}\right)^{-1}
$$

Again, iterative solution algorithm similar to that in [18] can be used to write a MATLAB program to solve for (52)-(55).

\section{Numerical Examples}

In this section, two numerical examples are provided to verify the proposed explicit model-following and implicit model-following designs in RSS form.

\subsection{Example for Explicit Model Following Design}

For the following unstable RSS system:

$$
x^{\prime}=A^{\prime} \dot{x}^{\prime}+B^{\prime} u=\left[\begin{array}{cc}
2.222 & -2.222 \\
11.1111 & -1.1111
\end{array}\right] \dot{x}^{\prime}+\left[\begin{array}{cc}
0.7778 & 2.2222 \\
3.3889 & 1.1111
\end{array}\right] u
$$

Note that the open loop poles are the reciprocals of the eigenvalues of matrix $A^{\prime}$, which are $0.025 \pm 0.2107 i$. Therefore, this system is unstable and will be diverge due to any none-zero initial condition if it is not properly controlled.

The system measurement output is state derivative related and is given as follows.

$$
y^{\prime}=C^{\prime} \dot{x}^{\prime}=\left[\begin{array}{ll}
1 & 1
\end{array}\right] \dot{x}^{\prime}
$$

If we have the following state derivative related performance output.

$$
Z=H \dot{x}^{\prime}=\left[\begin{array}{ll}
1 & 0.5
\end{array}\right] \dot{x}^{\prime}
$$

Suppose that the matrices of desired model specified in (8) are chosen as follows.

$$
\underline{A}=\left[\begin{array}{cc}
-1.3333 & -2.6667 \\
1.3333 & -1.3333
\end{array}\right], \underline{C}=\left[\begin{array}{ll}
1 & 0 \\
0 & 1
\end{array}\right] \text { and } \underline{H}=\left[\begin{array}{ll}
1 & 0.5
\end{array}\right]
$$

Note that the system poles of desired model are at $-0.25 \pm 0.3536 i$.

The initial stabilized feedback gain can be found using pole placement, eigenstructure assignment or other available design methods for output feedback control. 
Here the initial stabilized feedback gain for augmented system specified in (10) is selected as

$$
K_{0}=\left[\begin{array}{lll}
0.5 & 0 & 0 \\
0.5 & 0 & 0
\end{array}\right]
$$

The weighting matrices in (11) are selected as

$$
Q^{\prime}=0.1, \quad R=\left[\begin{array}{cc}
0.008 & 0 \\
0 & 0.05
\end{array}\right]
$$

The initial condition for the augmented system in (10) is given as

$$
x_{0}^{\mathrm{T}}=\left[\begin{array}{llll}
1 & 1 & 0.1 & 0.01
\end{array}\right]
$$

Iterative solution algorithm in [18] was adopted to write a MATLAB program to obtain the optimal state derivative feedback gain $K$ for the augmented system in (10) as follows.

$$
K=\left[\begin{array}{ccc}
1.8035 & -1.4630 & 0.2046 \\
0.8232 & -3.2809 & -1.5355
\end{array}\right]
$$

With this feedback gain $K$, the closed loop poles of the augmented system are now located at $-0.1500 \pm 0.1015 i$ and $-0.25 \pm 0.3536 i$.

Note that the original unstable poles at $0.025 \pm 0.2107 i$ were driven toward to the poles of the ideal model at $-0.25 \pm 0.3536 i$ and finally landed on stable locations at $-0.1500 \pm 0.1015 i$.

Given another initial condition of $x_{0}^{\mathrm{T}}=\left[\begin{array}{llll}1 & 1 & 1 & 1\end{array}\right]$, Figure 1 is the performance output response based on above explicit model-following design. We can find that the closed loop system does become stable. The error response between system's and model's performance outputs is given in Figure 2. We can find that the error does vanish with time. Therefore, the design goal is basically achieved.

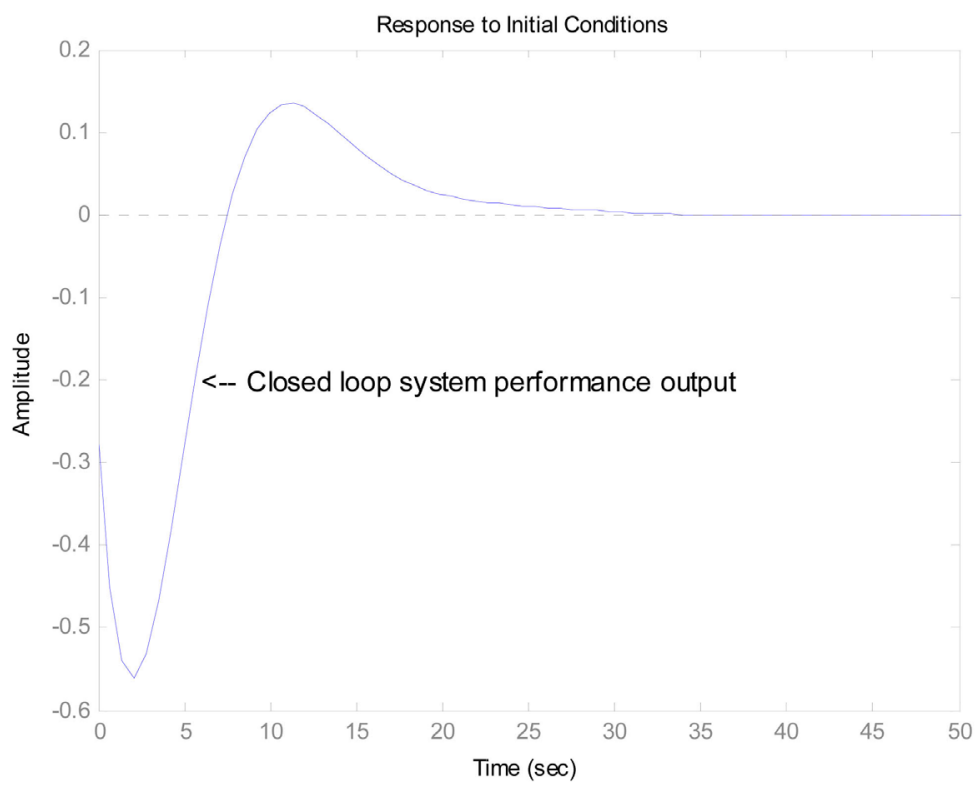

Figure 1. Performance output of explicit model-following design. 


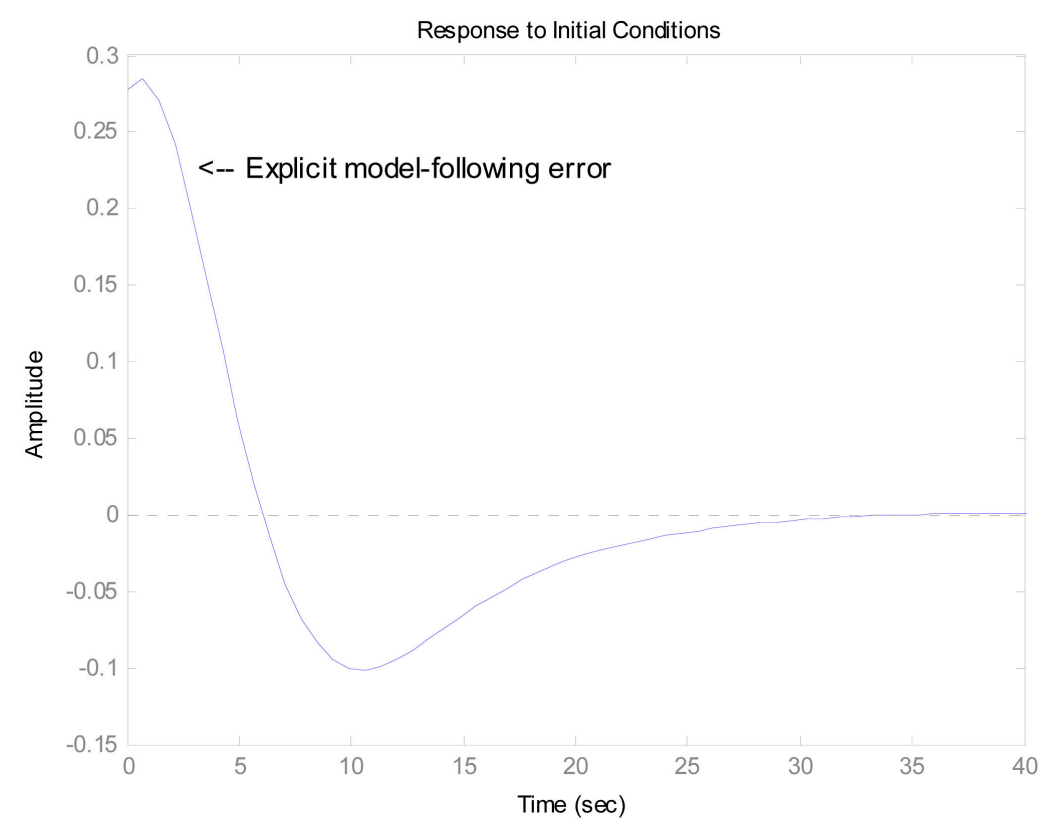

Figure 2. Error dynamics between system and model's performance outputs.

\subsection{Example for Implicit Model Following Design}

The same RSS system in example 4.1 is used to verify the validity of proposed implicit model following design algorithm except the performance out is state related as follows.

$$
Z=H x=\left[\begin{array}{ll}
1 & 0.5
\end{array}\right] x
$$

The matrix $\underline{A}$ of ideal model specified in (32) can only be chosen as a $1 \times 1$ constant because the number of performance output is only 1 . Therefore, we only can choose a real number. If -0.5 is the desired closed loop pole, the model system matrix is selected as

$$
\underline{A}=\frac{1}{-0.5}=-2
$$

The initial stabilized feedback gain is selected as follows.

$$
K_{0}=\left[\begin{array}{l}
0.5 \\
0.5
\end{array}\right]
$$

Applying initial stabilized feedback gain, system has stable poles at $-0.0432 \pm 0.1757 i$.

The weighting matrices in (38) are selected as

$$
Q=3, \quad R=\left[\begin{array}{cc}
0.1 & 0 \\
0 & 0.3
\end{array}\right]
$$

The initial condition for system is selected as

$$
x_{0}^{\mathrm{T}}=\left[\begin{array}{ll}
0.1 & 0.1
\end{array}\right]
$$

Iterative solution algorithm in [18] was adopted to write a MATLAB program to obtain the optimal state derivative feedback gain $K$ as follows. 


$$
K=\left[\begin{array}{l}
2.5371 \\
0.4899
\end{array}\right]
$$

Applying this optimal feedback gain, the closed loop poles of the system are driven toward to the poles of the ideal model and finally land on stable locations at -0.4720 and -0.1114 . Note that the closed loop pole at -0.4720 is indeed close to the ideal model pole at -0.5 .

Figure 3 is the performance output response based on above implicit model-following design due to different initial condition of $x_{0}^{\mathrm{T}}=\left[\begin{array}{ll}1 & 1\end{array}\right]$. From Figure 3, we can observe that the performance output can be further improved. That is because only one out of two eigenvalue was forced to follow the only one ideal model pole. Although the closed loop pole at -0.4720 is close to the ideal model pole at -0.5 , the other closed loop pole at -0.1114 downgraded the closed loop performance output.

Unlike the measurement output which is related to the number and locations of sensors in the system, performance output and ideal model only appear in performance index as design parameters and do not appear in the structure of implicit model-following controller. A designer has great freedom in selecting matrices $H$ in (32) and $\underline{A}$ in (34) for better closed loop performance. If state performance output $Z=H x=\left[\begin{array}{ll}1 & 0.5\end{array}\right] x$ is the most concerned in our design, we should keep it in a new $2 \times 2$ matrix which can allow us to assign a new $2 \times 2$ ideal model with two desired eigenvalues. Therefore, we can select a pair of complex conjugate as desired eigenvalues.

In design 2, the following new $H$ matrix is selected to improve closed loop performance.

$$
H=\left[\begin{array}{cc}
1 & 0.5 \\
-1 & 1
\end{array}\right]
$$

One can find that the first row of this matrix is the original $H$. Furthermore, a new $2 \times 2$ ideal model is selected as follows.

$$
\underline{A}=\left[\begin{array}{cc}
-1.3333 & -2.6667 \\
1.3333 & -1.3333
\end{array}\right]
$$

This ideal model has system poles at $-0.2500 \pm 0.3536 i$ which are the reciprocals of eigenvalues of $\underline{A}$. The new optimal state derivative feedback gain $K$ is then obtained as follows.

$$
K=\left[\begin{array}{l}
1.7317 \\
0.0411
\end{array}\right]
$$

Applying this optimal feedback gain, new closed loop poles are $-0.2309 \pm 0.1438 i$.

The first performance output in design 2 is exact the original performance output and it is shown in Figure 4. From the simulation result in Figure 4, we found that the performance output of design 2 is indeed better than that of design 1 . 


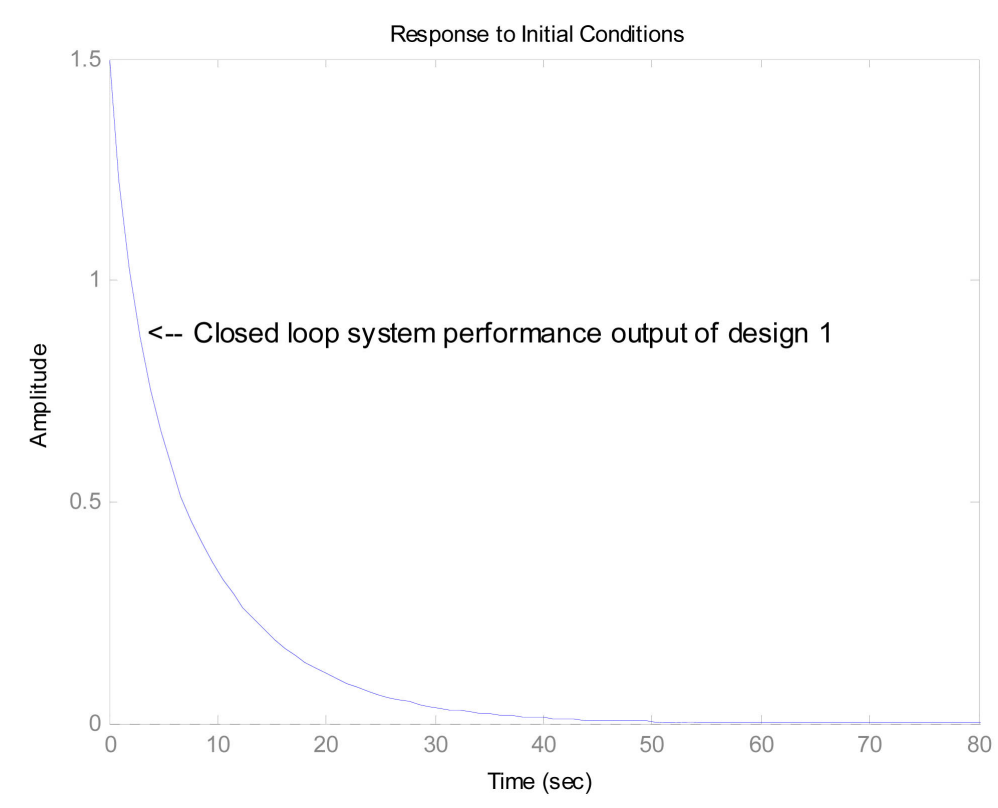

Figure 3. Performance output response of implicit model-following design 1.

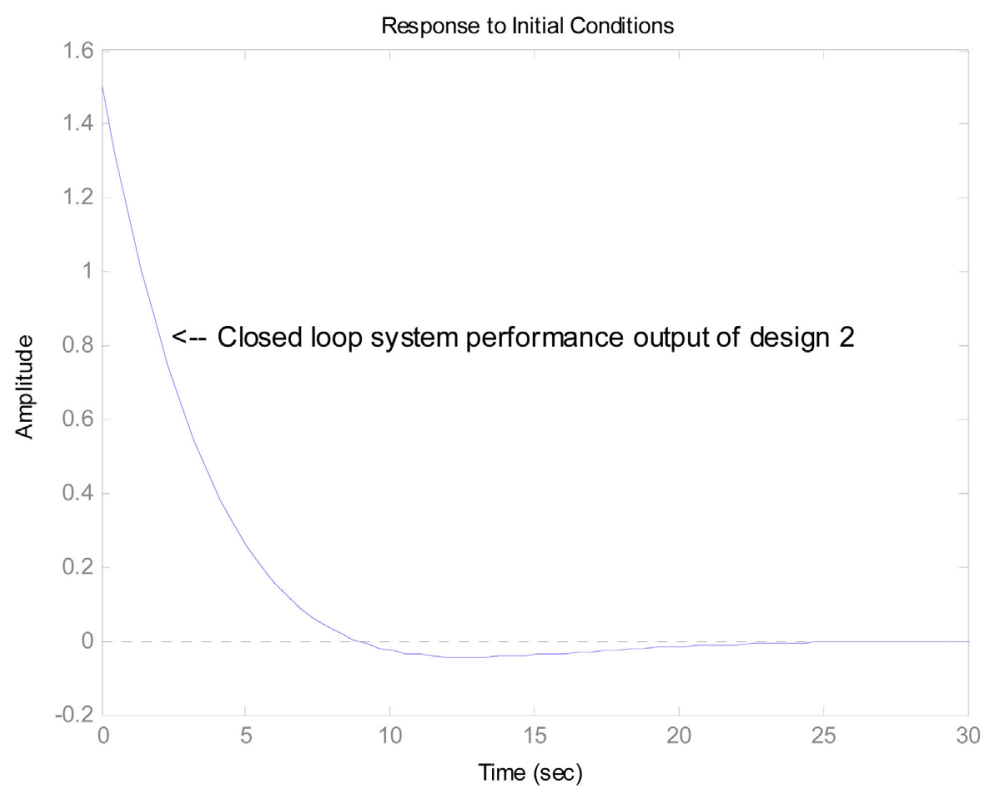

Figure 4. Performance output response of implicit model-following design 2.

\section{Conclusions}

The algorithms for both explicit and implicit model-following designs with state derivative measurement feedback in RSS form have been presented and the simulations have been carried out for verifications, too. The proposed explicit model-following design can handle the case of state derivative related performance output while implicit model-following design can handle the case of state related performance output. The proposed algorithms are analogous to their counterparts using state measurement feedback designs in state space form. Therefore, many design tools such as Lyapunov equation solver based on state 
space form can be adopted to carry out the proposed algorithms. For those who are familiar with state measurement feedback designs in standard state space form will have no difficulty to carry out the proposed algorithms in RSS form to deal with more problems that cannot be easily taken care of in state space form. Furthermore, a lot of mathematic overhead when people encounter in handling state derivative measurement feedback in generalized state space system form can also be avoided.

Although the purpose of model-following design is to drive the system to behave likes the ideal model, it is unlikely to get a closed loop system acts exactly like the ideal model. The reason is that the nature of model-following designs is output feedback design and only stability can be guaranteed. However, though adjusting design parameters such as ideal model matrix, performance matrix, weighting matrices, initial stabilized gain, error tolerance and initial condition, acceptable design will be found over the iterations.

Since micro-accelerometer is getting more and more attentions in many MEMS/NEMS applications and acceleration can only be modeled as state derivative, MEMS/NEMS applications utilizing state derivative feedback in RSS form are prospective.

\section{Acknowledgements}

Authors would like to thank the Ministry of Science and Technology, Taiwan for the financial support of this research under grant MOST 106-2221-E-214-008.

\section{Conflicts of Interest}

The authors declare no conflicts of interest regarding the publication of this paper.

\section{References}

[1] Campbell, S.L. (1982) Singular Systems of Differential Equations II. Pitman, Marshfield, Mass., USA.

[2] Newcomb, R.W. (1981) The Semistate Description of Nonlinear Time Variable Circuits. IEEE Transactions on Circuits and Systems, 28, 62-71. https://doi.org/10.1109/TCS.1981.1084908

[3] Brenan, K.E. (1986) Numerical Simulation of Trajectory Prescribed Path Control Problems by the Backward Differentiation Formulas. IEEE Transactions on Automatic Control, 31, 266-269. https://doi.org/10.1109/TAC.1986.1104236

[4] Tseng, Y.W. (2009) Vibration Control of Piezoelectric Smart Plate Using Estimated State Derivatives Feedback in Reciprocal State Space Form. International Journal of Control Theory and Applications, 2, 61-71.

[5] Pantelides, C.C. (1988) The Consistent Initialization of Differential Algebraic Systems. SIAM Journal on Scientific and Statistical Computing, 9, 213-231. https://doi.org/10.1137/0909014

[6] Kulah, H., Chae, J., Yazdi, N. and Najafi, K. (2006) Noise Analysis and Characterization of a Sigma-Delta Capacitive Microaccelerometer. IEEE Journal of Solid-State Circuits, 41, 352-361. https://doi.org/10.1109/JSSC.2005.863148 
[7] Verghese, G.C., Levy, B.C. and Kailath, T. (1981) A Generalized State Space for Singular Systems. IEEE Transactions on Automatic Control, 26, 811-831. https://doi.org/10.1109/TAC.1981.1102763

[8] Cobb, D. (2006) State Feedback Impulse Elimination for Singular Systems over a Hermite Domain. SIAM Journal on Control and Optimization, 44, 2189-2209. https://doi.org/10.1137/040618515

[9] Liu, D., Zhang, G. and Xie, Y. (2009) Guaranteed Cost Control for a Class of Descriptor Systems with Uncertainties. International Journal of Information \& Systems Sciences, 5, 430-435.

[10] Saadni, M.S., Chaabane, M. and Mehdi, D. (2006) Robust Stability and Stabilization of a Class of Singular Systems with Multiple Time Varying Delays. Asian Journal of Control, 8, 1-11. https://doi.org/10.1111/j.1934-6093.2006.tb00245.x

[11] Varga, A. (2000) Robust Pole Assignment for Descriptor Systems. Proceeding of the Mathematical Theory of Networks and Systems, Perpignan, 12-16 August 2000. https://core.ac.uk/download/pdf/11096417.pdf

[12] Cobb, D. (2010) Eigenvalue Conditions for Convergence of Singularly Perturbed Matrix Exponential Functions. SIAM Journal on Control and Optimization, 48, 4327-4351. https://doi.org/10.1137/09075113X

[13] Yeh, F.B. and Huang, H.N. (2000) H Infinity State Feedback Control of Smart Beam-Plates via the Descriptor System Approach. Tunghai Science, 2, 21-42.

[14] Tseng, Y.W. (2008) Control Designs of Singular Systems Expressed in Reciprocal State Space Framework with State Derivative Feedback. International Journal of Control Theory and Applications, 1, 55-67.

[15] Tseng, Y.W. and Wang, Y.N. (2013) Sliding Mode Control with State Derivative Output Feedback in Reciprocal State Space Form. Abstract and Applied Analysis, 2013, Article ID: 590524. https://www.hindawi.com/journals/aaa/2013/590524/ https://doi.org/10.1155/2013/590524

[16] Tseng, Y.W., Kwak, S.K. and Yedavalli, R.K. (2003) Stability, Controllability and Observability Criteria for the Reciprocal State Space Framework. Proceedings of the 2003 American Control Conference, Denver, 4-6 June 2003, 5093-5097. https://ieeexplore.ieee.org/document/1242535

[17] Tseng, Y.W. and Hsieh, J.G. (2013) Optimal Control for a Family of Systems in Novel State Derivative Space Form with Experiment in a Double Inverted Pendulum System. Abstract and Applied Analysis, 2013, Article ID: 715026. https://www.hindawi.com/journals/aaa/2013/715026/ https://doi.org/10.1155/2013/715026

[18] Stevens, B.L. and Lewis, F.L. (1992) Aircraft Control and Simulation. John Wiley and Sons, Inc., New York, 421-437.

[19] Kreindler, E. and Rothschild, D. (1976) Model-Following in Linear Quadratic Optimization. Journal of AIAA, 14, 835-842. https://doi.org/10.2514/3.7160 